\title{
Multiorganic fat embolism in a case of post-traumatic fracture of the humerus
}

\author{
Adriana Grigoras ${ }^{* 1,2}$, Laura Knieling ${ }^{1,2}$, Carmen Mateiciuc ${ }^{3}$, Irina Draga Caruntu ${ }^{1}$, \\ Cornelia Amalinei $i^{1,2}$
}

1 "Grigore T. Popa" University of Medicine and Pharmacy, lasi, ${ }^{2}$ Institute of Legal Medicine lasi,

${ }^{3}$ Legal Medicine Service Bacau, Romania

\begin{abstract}
Fat embolism syndrome is a known complication in traumatology, especially in long bone fractures. The incidence of the fat embolism syndrome depends on the bone involved, whether fractures are isolated or multiple, the age of the patient, and the gender. It rarely occurs as a result of medical conditions. Classically, patients presents with a triad consisting of pulmonary distress, mental status changes, and petechial rash, occurred 24 to 48 hours after long-bone fracture. We present the case of an 88 year old woman, a victim of a traffic accident (pedestrian), who died in the same day. Autopsy and microscopic examination of fragments of tissue stained with Sudan III confirmed the presence of a multiorganic fat embolism in this case of post-traumatic fracture of the humerus.
\end{abstract}

Keywords: fat embolism, autopsy, humerus fracture

\section{Introduction}

A fat embolism is represented by the migration of a fat particle from the bone's marrow to a blood vessel. This is caused by the fragmentation of the bone marrow, rich in lipids, due to fractures or surgery of the bone, especially the bones of the lower limbs or the pelvis, followed by its migration into the blood stream [1]. The clinical signs are evident after a few hours from the time of the operation or fracture; the signs are represented by fever, purpura, or acute respiratory deficiency.

Received: April 2014; Accepted after review: June 2014; Published: June 2014

${ }^{*}$ Corresponding author: Adriana Grigoras, "Grigore T. Popa" University of Medicine and Pharmacy, lași, Institute of Legal Medicine lasi, Romania. Email: a grigoras6600@yahoo.com
To these, one can add in various proportions, neuropsychiatric disorders with agitation, confusion, or coma. Fat embolisms have a $15-30 \%$ mortality rate.

However, it is not certain if the embolism itself or the context in which it was produced is responsible for its high rate of mortality. In some cases, embolisms can be cured without any side effects in approximately 2 weeks [2, 3]

\section{Material and Method}

The case is of an 88 year-old woman, a victim of a road accident (pedestrian) that was thereafter transferred to the emergency room at the county hospital in Bacau. There, after clinical and laboratory exams were done, she was diagnosed with a closed left humeral buckle and transverse fracture. The abdominal echographic examination showed no post 
traumatic injuries to any internal organs, such as the liver, spleen, and kidneys. After an orthopedic reduction of the fracture, the patient had been admitted to a specialized clinic. The family refused admission and thus, the patient was transferred to her domicile, where, she later passed away that day. Following her death, a necropsy had taken place and fragments of brain, heart, lung, and kidney tissue were harvested for microscopic examination. The different tissue fragments were treated differently as to obtain frozen slices of tissue, stained using the Sudan III method, as well as in the classical method by inclusion in paraffin and later to be sliced and stained using hematoxylin-eosine (H.E.). The microscopic examination was done by using a Leica microscope.

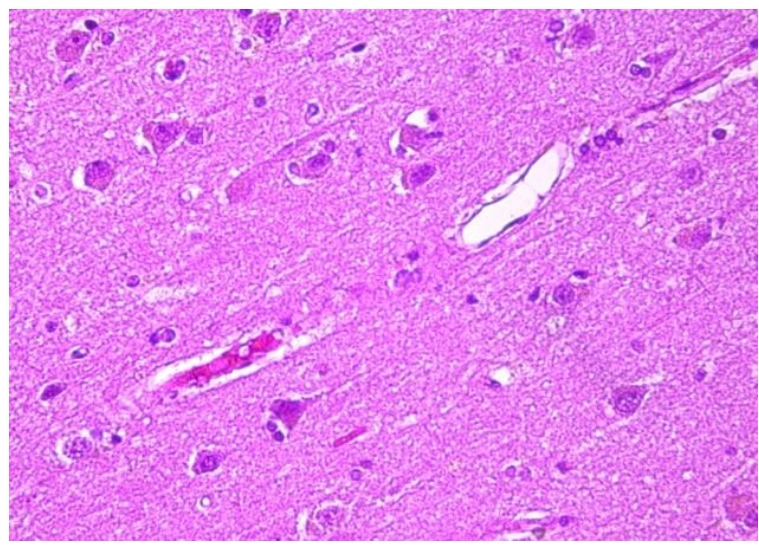

Fig. 1. An empty vacuole that occupies the entire lumen of an intracerebral blood vessel, H.E. X 200

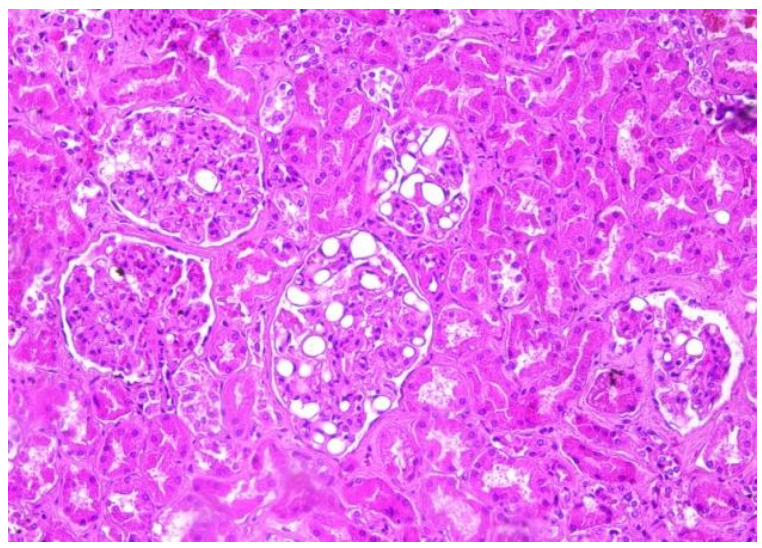

Fig. 3. Glomerular capillaries with empty vacuoles, H.E. $\times 200$

\section{Results}

The necropsy confirmed the presence of a closed displaced fracture of the left humerus with a confirmed pre-existing pathology that comes with age such as: lesions of cerebral atherosclerosis, myocardial fibrosis, pancreatic sclerosis, calcified thoracoabdominal aortic atheromatosis, nephrosclerosis, and degradation of the liver. The microscopic examination of the tissular fragments stained with H.E. showed the presence of empty vacuoles in the small caliber intracerebral vascular lumens (Fig. 1, 2), glomerular capillaries of the renal corpuscles (Fig. 3, 4), and pulmonary interalveolar septal capillaries (Fig. 5, 6). These emboli occupied a variable percent of the luminal diameter, and were clearly identified if the blood vessels also contained blood.

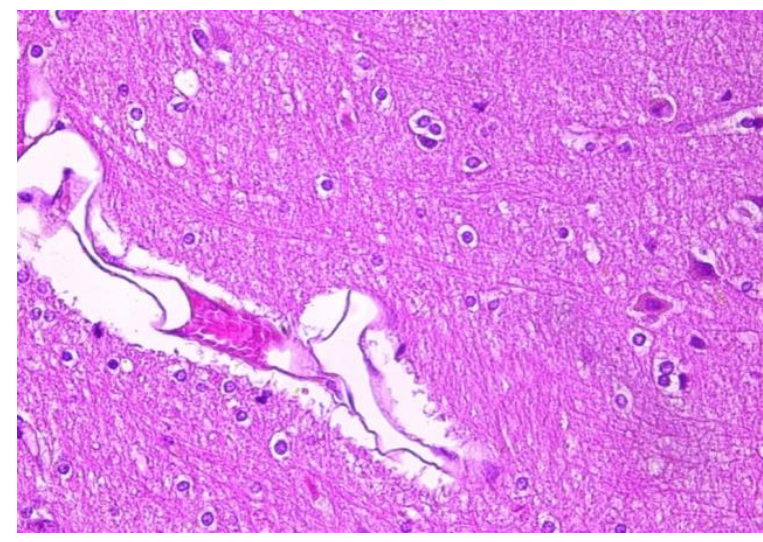

Fig. 2. An empty vacuole that partially occupies the lumen of an intracerebral blood vessel, H.E. x 200

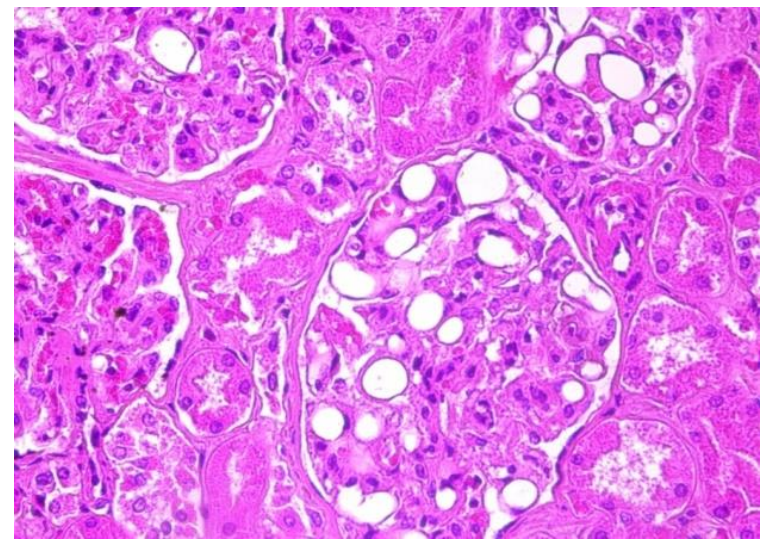

Fig. 4. Detail of Fig. 3, H.E. x 400 


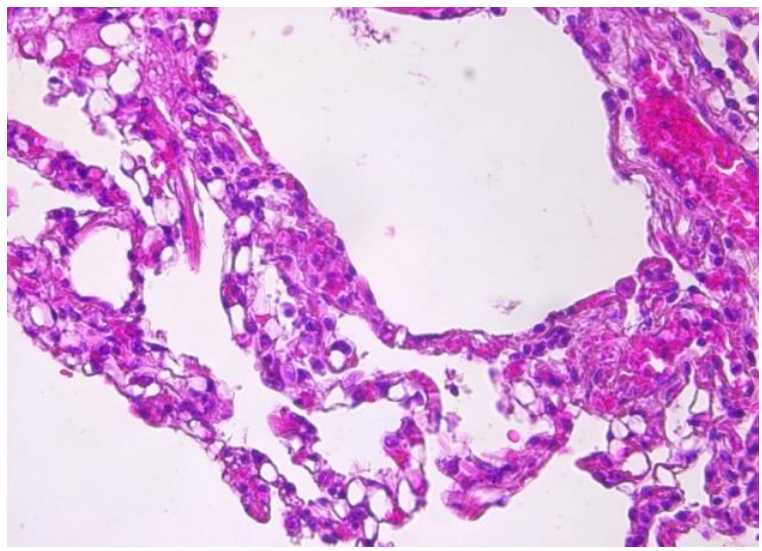

Fig. 5. Images of empty vacuoles in smaller caliber blood vessels in interalveolar pulmonary septa, H.E. $x$ 200

The examination of the frozen sections clearly demonstrated the lipid nature of these intravascular vacuoles. The microscopic images of these vacuoles overlapped the

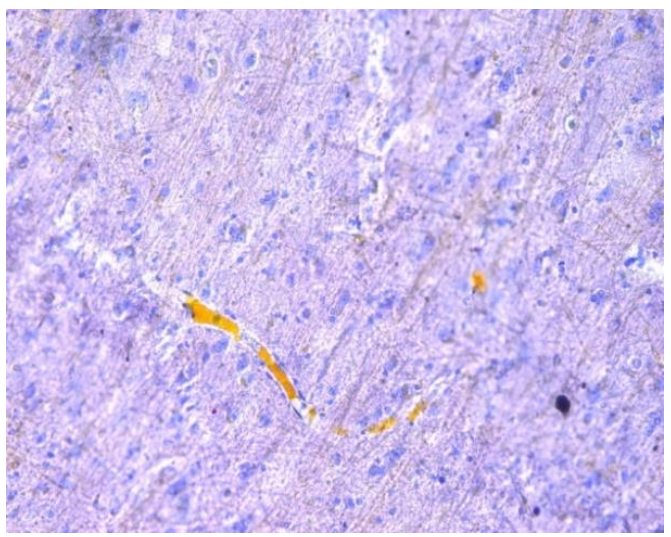

Fig. 7. Lipid content that partially occupies the lumen of an intracerebral blood vessel, Sudan III $x$ 200

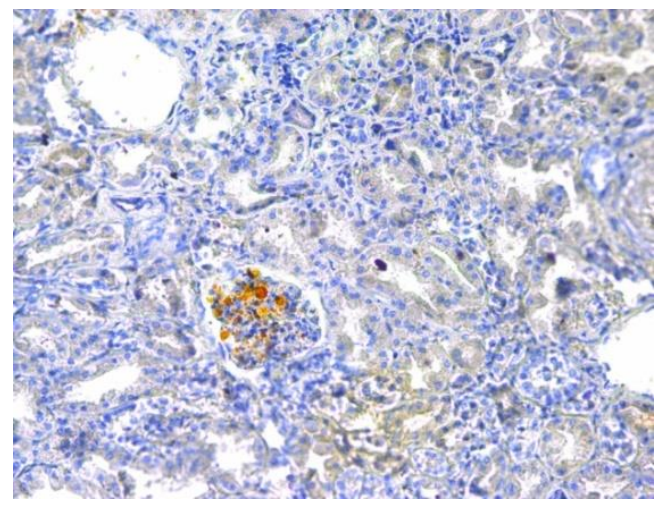

Fig. 9. Lipid content in the glomerular capillaries of a renal corpuscle, Sudan III x 200

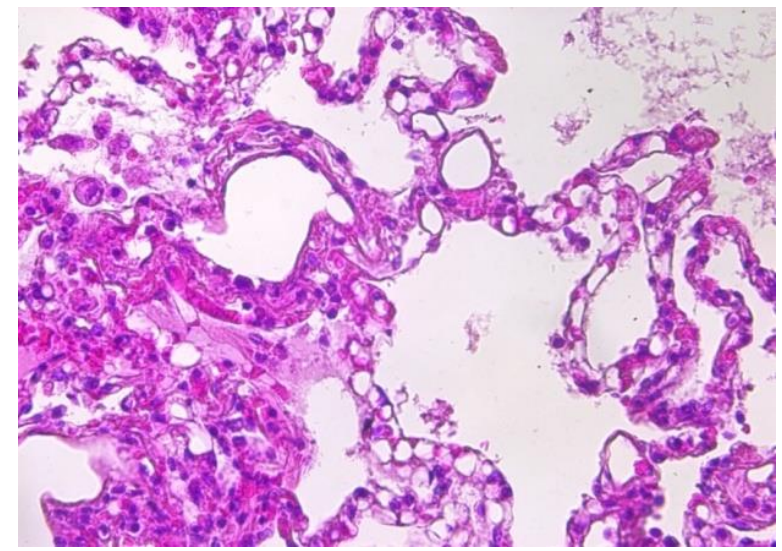

Fig. 6. Numerous empty vacuoles in interalveolar pulmonary septa, H.E. x 200

lesions stained by H.E. in cerebral (Fig. 7, 8), renal (Fig. 9, 10), and pulmonary (Fig. 11, 12) tissue.

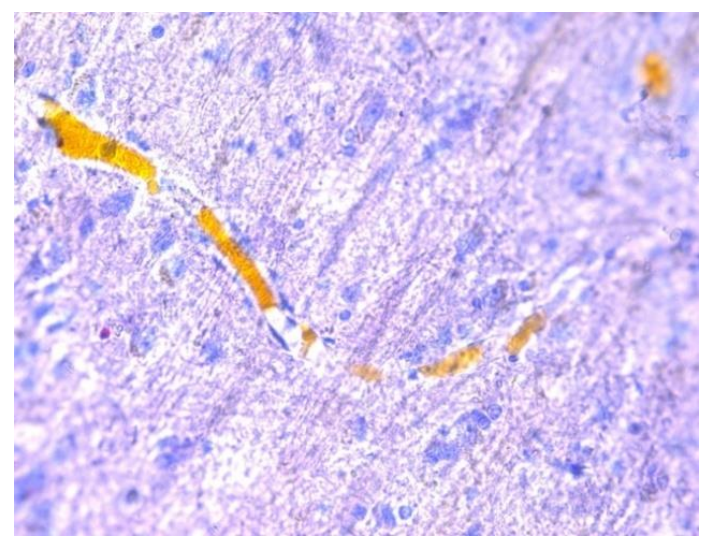

Fig. 8. Detail of fig. 7, Sudan III x 400

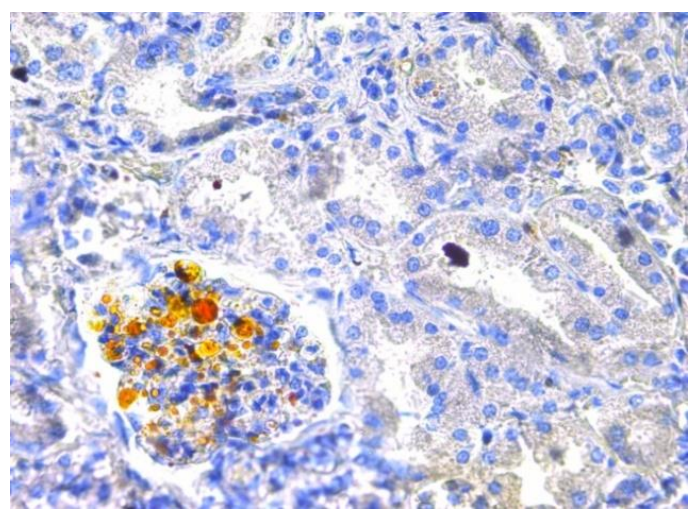

Fig. 10. Detail of fig. 8, Sudan III x 400 


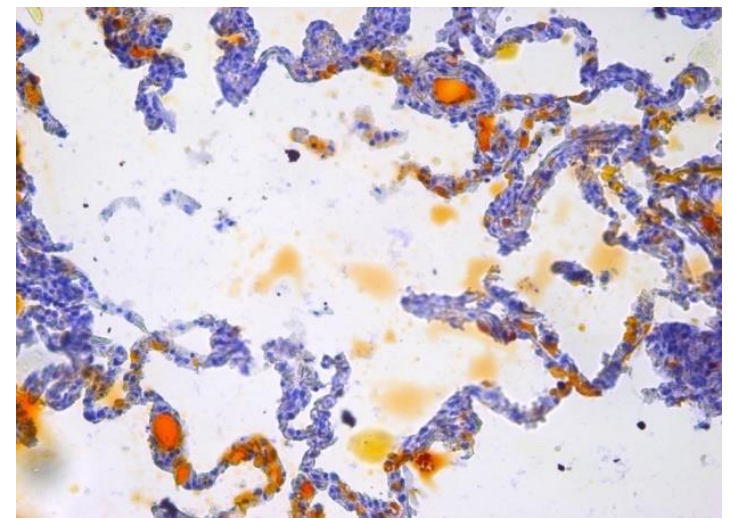

Fig. 11. Blood vessels of the interalveolar septa with intraluminal lipid content, Sudan III x 200

\section{Discussion}

A fat embolism was first described in 1862 , in medical literature pertaining to this specialty, by Zenker, during a necropsy. In 1873, Von Bergmann described the clinical signs associated with the post metabolic syndrome containing lipids [2, 4].

However, these clinical signs remain underdiagnosed, the symptomatology of the embolic syndrome with lipid material being nonspecific. The triggering event of a fat embolism can be a traumatism or surgery. The mortality rate is higher in patients that without an associated chronic pathology, which can, at times, become acute within this context $(5,6)$. In medical literature, this syndrome is not frequently observed when one analyses an isolated fracture of the humerus, as presented in this case [7].

The clinical aspects are nonspecific and can be noted as neurologic manifestations of a nonspecific etiology, such as irritability and agitation, which can progress to coma. 20-50\% of cases may be associated with purpura without thrombocytopenia and with respiratory symptoms, such as dyspnea and tachypnea [8]. These major symptoms are associated with fever (above $38.5^{\circ} \mathrm{C}$ ), tachycardia, or other dysfunctions correlated to renal failure. Furthermore, patients can have anemia, due to aggregation and hemolysis of the red blood cells. In the present case, in the absence of

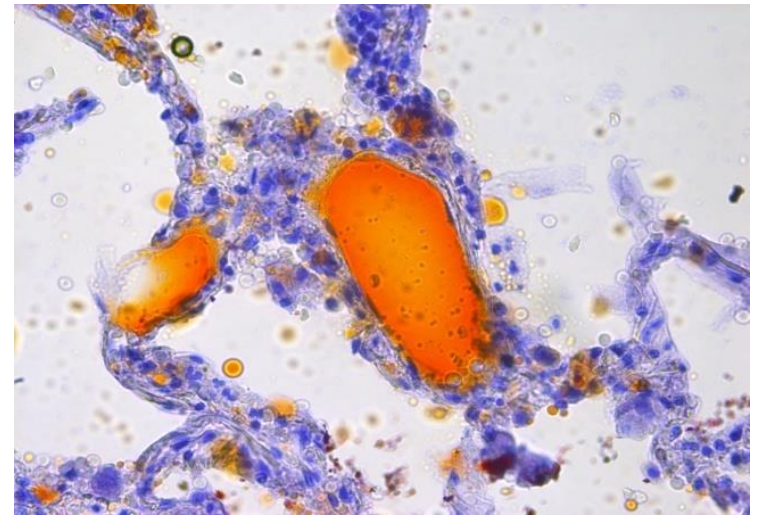

Fig. 12. Blood vessels of the interalveolar septa with intraluminal lipidic content, Sudan III x 400

medical assistance, following patient refusal of admittance to the hospital and appropriate treatment, the triggering event has been the catalyst that led to patient's death.

The pathogenesis of the fat embolic syndrome involves a as well as a biochemical mechanism [9]. Post traumatically, large lipid droplets are released from the bone marrow or from the injured adipose tissue within the circulatory system. These emboli reach the pulmonary capillary bed via venous system and brain, due to arterial-venous shunts. Lipid microemboli cause an initial mechanical blockage of the small intracerebral and pulmonary blood vessels, which is followed by a local exacerbation of aggregated red and white blood cells. Moreover, free fatty acids provoke vascular endothelial lesions which in turn, result in vascular permeability increase with the onset of pulmonary edema [9].

As clinical and paraclinical manifestation provide only nonspecific data the diagnosis is difficult. From all the data collected, some are highly suggestive, such as: low hematocrit value in 23-48 hours after the initial lesion due to intra-alveolar microhemorrhages and thrombocytopenia as well as modified clotting tests.

Although histopathology is the only method which can uphold a diagnostic it is reserved to postmortem examination, performed on tissue fragments stained with dyes that have an affinity for lipids, such as Sudan III or IV [6, 9]. 


\section{Conclusions}

The microscopic examination confirmed the triggering event of the patient's death consisting in a fracture of the left humerus

\section{References}

1. Stein PD, Yaekoub AY, Matta F, et al.: Fat embolism syndrome. Am J Med Sci, 2008, 336(6):472-7.

2. Taviloglu , Yanar H: Fat Embolism Syndrome. Surgery Today, 2007, 37(1):5-8.

3. Mellor A, Soni N: Fat embolism. Anaesthesia, 2001, 56(2):145-54.

4. Parisi DM, Koval K, Egol K: Fat embolism syndrome. Am J Orthop, 2002, 31(9):507-12.

5. Nikolić S, Micić J, Savić S, et al.: Post-traumatic systemic fat embolism syndrome. Retrospective autopsy study. Srp Arh Celok Lek, 2000, 128 $(1-2): 24-8$. bone complicated with a multiorganic fat embolism (involving brain, lung, and kidneys), which developed on a background of agerelated pathology.

6. Zhou L, Mu J, Dong HM, et al.: Forensic analysis of death caused by fat embolism: A study of 20 autopsy cases. Fa Yi Xue Za Zhi, 2013, 29(6):431-3.

7. Russell GV Jr, Kirk PG, Biddinger P: Fat embolism syndrome from an isolated humerus fracture. Orthop Trauma, 1997, 11(2):141-4.

8. van den Brand JG, van der Hoeven JH, Olsman JG: Dyspnea or confusion after trauma? Consider fat embolism syndrome. Ned Tijdschr Geneeskd, 2000, 144(32):1513-7.

9. Kumar V, Abbas AK, Fausto N: Robbins Basic Pathology. 8th edition. Edited by Mitchell RN. Saunders Elsevier publishing, 2007, 505-506 